\title{
Stability of fluid in a rectangular enclosure by spectral method
}

\author{
N. Y. LEE and W. W. SCHULTZ \\ Department of Mechanical Engineering and Applied Mechanics, University of Michigan, \\ Ann Arbor, MI 48109, U.S.A. \\ and
}

J. P. BOYD

Department of Atmospheric, Oceanic and Space Sciences, University of Michigan, Ann Arbor, MI 48109, U.S.A.

(Received 21 March 1988 and in final form 29 July 1988)

\begin{abstract}
The hydrodynamic stability of Rayleigh convection in a rectangular cavity is studied numerically. The calculation assumes that the Boussinesq fluid motion is two-dimensional. The characteristic value equations are solved by a Chebyshev spectral method. Care must be taken when obtaining the equivalent algebraic eigenproblem to avoid spurious roots and inaccurate results. Critical Rayleigh numbers are determined for various aspect ratios.
\end{abstract}

\section{INTRODUCTION}

THE RAYLEIGH convection problem is often considered a paradigm for the study of transition to turbulence. Computational models of this problem must have very high spatial resolution and hence are usually spectral. Chebyshev spectral methods for the solution of hydrodynamic stability eigenvalue problems have been developed by Orszag [1]. The numerical results [1 4 ] illustrate that much greater accuracy is achieved by Chebyshev expansions than by expansions in other orthogonal functions. They often require periodic boundary conditions in one or two directions as a computational convenience. To avoid the difficulties associated with wave number selection and to make adequate comparisons with experiments, we consider Rayleigh convection in a box. Unfortunately, the box corners introduce singularities for fluid flow problems, which destroy the exponential convergence properties of spectral methods.

When natural convection occurs in an infinite layer bounded by two horizontal planes, studies of the nonlinear system show that two-dimensional convection in rolls is stable [5]. When the fluid is confined by side walls, the first bifurcation produces three-dimensional, steady rolls with the horizontal velocity in the third direction [6]. Davis [6] considered the threedimensional flow for perfectly conducting side walls. However, Kurzweg [7] studied fluid stability for twodimensional natural convection in a rectangular enclosure with insulated side walls. Velte [8] examined the same geometry with perfect conducting walls.

In this paper we reconsider the problem of the stability of a two-dimensional rectangular box of fluid heated from below for both conducting and insulating side walls. While this model is not physical because we prohibit flow in the third direction, the methods we describe can be extended to three-dimensional flows and eventually to the study of transition to turbulence. Our objective is to demonstrate more accurate solutions using Chebyshev spectral methods than those computed previously by other techniques. The critical Rayleigh numbers are determined for various aspect ratios and compared to previous results. We examine the convergence and how it is affected by the corner singularities.

\section{FORMULATION}

We consider the motion of a Boussinesq fluid in a two-dimensional rectangular cavity. The direction of gravity is along the $y$-axis and the fluid is motionless. The base of the rectangle is fixed at a temperature higher than the top. Conditions in the basic state are characterized by zero velocity, a constant temperature gradient, and a constant pressure gradient caused by gravity. An infinitesimal disturbance of the initially quiescent layers is assumed, and non-linear terms are neglecled. For the Boussinesq approximation, the differential equations and boundary conditions represent a self-adjoint system, guaranteeing that terms with time derivatives will not appear in the equations governing the perturbations at the point of marginal stability $[6,9]$. The length scales of the half-height for the $y$-direction and half-width for the $x$-direction are used with a coordinate system in the center of the box so that Chebyshev polynomials can be directly applied. Conservation of linear momentum and energy lead to the following set of non-dimensional equations of the marginal stability state: 


\section{NOMENCLATURE}

$A \quad$ aspect ratio

$a_{n m}$ expansion coefficients for $\psi$

$b_{n m}$ expansion coefficients for $\theta$

Bi Biot number

$D \quad$ width of cavity

$g$ acceleration of gravity

$H$ height of cavity

$h \quad$ heat transfer coefficient

$k_{\mathrm{s}} \quad$ thermal conductivity of the solid wall

$N, M, P, Q$ truncation for spectral coefficients

$R a \quad$ Rayleigh number

$R a_{\mathrm{c}} \quad$ critical Rayleigh number

$\Delta T$ temperature difference, $T_{\mathrm{h}}-T_{\mathrm{c}}$
$U, V$ expansion functions for $\psi$

$X, Y$ expansion functions for $\theta$

$x$ dimensionless horizontal coordinate

$y$ dimensionless vertical coordinate

$x_{i}, y_{i}$ collocation points

$w \quad$ Chebyshev weight function.

Greek symbols

$\alpha \quad$ thermal coefficient of expansion

$\Gamma, \Lambda, \Upsilon, \Delta$ integrals defined in equations ( 7$)$

0 temperature perturbation

$\kappa \quad$ thermal diffusivity

$v \quad$ kinematic viscosity

iv stream function.

$$
\begin{aligned}
& \nabla^{4} \psi=\frac{A^{3}}{8} R a \frac{\partial \theta}{\partial x} \\
& \nabla^{2} \theta=\frac{A}{2} \frac{\partial \psi}{\partial x}
\end{aligned}
$$

where

$$
\nabla^{2} \equiv \frac{\partial^{2}}{\partial x^{2}}+A^{2} \frac{\partial^{2}}{\partial y^{2}}
$$

is the scaled Laplacian operator and $A$ the lengthto-height aspect ratio $(D / H)$. The two-dimensional continuity requirement is satisfied by $\psi$, the perturbation stream function and $\theta$ is the perturbation temperature. The boundary conditions require zero velocity at all walls and conducting $(B i=0)$ or insulating $(B i=\infty)$ side walls such that

$$
\psi=\frac{\partial \psi}{\partial y}=\theta=0 \quad \text { on } y= \pm 1
$$

and

$$
\psi=\frac{\partial \psi}{\partial x}=\theta+B i \frac{\partial \theta}{\partial x}=0 \quad \text { on } x= \pm 1
$$

Here, $B i$ is the Biot number and $R a$ the Rayleigh number defined in the usual way

$$
B i=\frac{h H}{k_{\mathrm{s}}}
$$

and

$$
R a=\frac{\alpha \Delta T g H^{3}}{v \kappa}
$$

\section{METHOD OF SOLUTION}

The governing equations and boundary conditions constitute an eigenvalue problem for the Rayleigh number. The lowest eigenvalue is the desired critical Rayleigh number. To solve the characteristic value equations for arbitrary aspect ratio, the dependent variables can be expanded in double-truncated series of basis functions that satisfy boundary conditions (1c) and (1d). The tau method is not used because it does not lead to a standard form of the algebraic eigenvalue problem. The eigenfunction expansions are then given by

$$
\begin{aligned}
& \psi=\sum_{n=1}^{N} \sum_{m=1}^{M} a_{n+m} U_{n}(x) V_{m}(y) \\
& \theta=\sum_{p=1}^{p} \sum_{q=1}^{Q} b_{p q} X_{p}(x) Y_{q}(y)
\end{aligned}
$$

where $a_{n m}$ and $b_{p q}$ are the expansion coefficients, and $U, V, X$, and $Y$ are one-dimensional basis functions for stream function and temperature. Symmetry properties greatly reduce the size of the algebraic system since system (1) may be solved using only those basis functions that have the same symmetry as the solution. Physically relevant sets of expansion functions that satisfy boundary conditions and do not interact with each other fall into two classes. The basis functions for an odd number of rolls in the horizontal direction are

$$
U_{n}(x)=T_{2 n+2}(x)-(n+1)^{2} T_{2}(x)+\left(n^{2}+2 n\right) T_{0}(x)
$$

$V_{m}(y)=T_{2 m+2}(y)-(m+1)^{2} T_{2}(y)+\left(m^{2}+2 m\right) T_{0}(y)$

$$
Y_{u}(y)=T_{2 y}(y)-T_{0}(y)
$$

and

$$
X_{p}(x)=T_{2 p+1}(x)-T_{1}(x)
$$

$$
\text { (conducting sides, } B i=0 \text { ) }
$$

or

$$
X_{p}(x)=T_{2 p+1}(x)-(2 p+1)^{2} T_{1}(x)
$$

(insulated sides, $B i=\infty$ ). 
The basis functions for an even number of rolls in the horizontal direction are

$$
\begin{aligned}
U_{n}(x)=T_{2 n+3}(x)-\frac{1}{2}(n+1)(n & +2) T_{3}(x) \\
& +\frac{1}{2} n(n+3) T_{1}(x)
\end{aligned}
$$

and

$$
\begin{array}{r}
X_{p}(x)=T_{2 p}(x)-T_{0}(x) \\
\quad \text { (conducting sides, } B i=0)
\end{array}
$$

or

$$
\begin{aligned}
X_{p}(x)=T_{2 p+2}(x)-(p+1)^{2} T_{2}(x) & \\
& \text { (insulated sides, } B i=\infty)
\end{aligned}
$$

with the same basis functions in the $y$-direction. We do not consider odd basis functions in the $y$-direction because the most unstable eigenvalues have only one cell in the vertical direction, regardless of the aspect ratio.

Substituting equations (2) into equations (1a) and (1b) gives

$$
\begin{aligned}
& \sum_{n=1}^{N} \sum_{m=1}^{M} a_{n m} \nabla^{4} U_{n}(x) V_{m}(y) \\
&- \frac{A^{3}}{8} R a \sum_{p=1}^{P} \sum_{q=1}^{Q} b_{p q} \frac{\partial X_{p}}{\partial x} Y_{q}(y)=0 \\
&-\frac{A}{2} \sum_{n=1}^{N} \sum_{m=1}^{M} a_{n m} \frac{\partial U_{n}}{\partial x} V_{m}(y) \\
&+\sum_{p=1}^{P} \sum_{q=1}^{Q} b_{p q} \nabla^{2} X_{p}(x) Y_{q}(y)=0
\end{aligned}
$$

The Galerkin equations are obtained by multiplying equation (5a) by $U_{k}(x) V_{l}(y)$ and equation (5b) by $X_{k}(x) Y_{l}(y)$ and then integrating over the range $-1 \leqslant x \leqslant 1$ and $-1 \leqslant y \leqslant 1$ with the usual Chebyshev weight function [10]. The standard procedure of forming the algebraic system uses inner products with Chebyshev polynomials, i.e. multiplying equation (5a) by $T_{k}(x) T_{i}(y)$ and equation (5b) by $T_{k}(x) T_{l}(y)$ with the same integration weight and interval [1]. The use of a true Galerkin method rather than the standard procedure is important since its use ensures that the algebraic system is also self-adjoint and it avoids the problems of complex eigenvalues and spurious roots [10]. In Section 4 we briefly discuss the poor convergence properties when the inner product with Chebyshev polynomials is used. Our Galerkin procedure requires that

$\left|\begin{array}{ccccc}\Gamma_{11}^{11} & \Gamma_{12}^{11} \ldots \Gamma_{N M}^{11} & R a \Lambda_{11}^{11} & R a \Lambda_{12}^{11} \ldots R a \Lambda_{P Q}^{11} \\ \Gamma_{11}^{12} & \Gamma_{12}^{12} \ldots \Gamma_{N M}^{12} & R a \Lambda_{11}^{12} & R a \Lambda_{12}^{12} \ldots R a \Lambda_{P Q}^{12} \\ \vdots & \ddots & \vdots & \ddots \\ \Gamma_{11}^{N M} & \Gamma_{12}^{N M} \ldots \Gamma_{N M}^{N M} & R a \Lambda_{11}^{N M} & R a \Lambda_{12}^{N M} \ldots R a \Lambda_{P Q}^{N M} \\ \Upsilon_{11}^{11} & \Upsilon_{12}^{11} \ldots \Upsilon_{N M}^{11} & \Delta_{11}^{11} & \Delta_{12}^{11} \ldots \Delta_{P Q}^{11} \\ \Upsilon_{11}^{12} & \Upsilon_{12}^{12} \ldots \Upsilon_{N M}^{12} & \Delta_{11}^{12} & \Delta_{12}^{12} \ldots \Delta_{P Q}^{12} \\ \vdots & \ddots & \vdots & \ddots \\ \Upsilon_{11}^{P Q} & \Upsilon_{12}^{P Q} \ldots \Upsilon_{N M}^{P Q} & \Delta_{1 Y}^{P Q} & \Delta_{12}^{P Q} \ldots \Delta_{P Q}^{P Q}\end{array}\right|=0$

where using similar nomenclature to Kurzweg [7], $\Gamma_{n m}^{k l}, \Lambda_{p q}^{k l}, \Upsilon_{n m}^{k l}$, and $\Delta_{p q}^{k l}$ are two-dimensional arrays formed by

$$
\begin{aligned}
\Gamma_{n m}^{k l} & =\int_{-1}^{1} \int_{-1}^{1} U_{k} V_{l} \nabla^{4}\left(U_{n} V_{m}\right) w(x, y) \mathrm{d} x \mathrm{~d} y \\
\Lambda_{p q}^{k l} & =-\frac{A^{3}}{8} \int_{-1}^{1} \int_{-1}^{1} U_{k} V_{l} \frac{\partial X_{p}}{\partial x} Y_{q} w(x, y) \mathrm{d} x \mathrm{~d} y \\
\Upsilon_{n m}^{k l} & =-\frac{A}{2} \int_{-1}^{1} \int_{-1}^{1} X_{k} Y_{l} \frac{\partial U_{n}}{\partial x} V_{m} w(x, y) \mathrm{d} x \mathrm{~d} y \\
\Delta_{p q}^{k l} & =\int_{-1}^{1} \int_{-1}^{1} X_{k} Y_{l} \nabla^{2}\left(X_{p} Y_{q}\right) w(x, y) \mathrm{d} x \mathrm{~d} y
\end{aligned}
$$

where $w(x, y)=\left(1-x^{2}\right)^{-1 / 2}\left(1-y^{2}\right)^{-1 / 2}$ is the twodimensional Chebyshev weight function. We have chosen to represent the temperature and stream function by the same truncation, $N=P$ and $M=Q$. Equations (7a)-(7d) are integrated using the inner products for the Chebyshev polynomials listed in the Appendix. The determinant (6) can easily be converted into the standard form

$$
\mathbf{A} x=R a \mathbf{B} x .
$$

Solutions are then obtained from the EISPACK: RGG subroutine, which uses the $Q Z$-algorithm.

Alternatively, we obtain the algebraic eigenvalue problem from the pseudospectral method [11, 12], using collocation points that are roots of the Chebyshev polynomials

$$
\begin{aligned}
& x_{i}=-\cos \left(\begin{array}{c}
\pi(2 i-1) \\
4 N
\end{array}\right), \quad i=1, \ldots, N \\
& y_{j}=-\cos \left(\frac{\pi(2 j-1)}{4 M}\right), \quad j=1, \ldots, M .
\end{aligned}
$$

All the collocation points lie in $-1 \leqslant x \leqslant 0$ and $-1 \leqslant y \leqslant 0$ because of the symmetries. The basis functions are again equations (3) and (4) which satisfy the boundary conditions. The pseudospectral algebraic system is formed by requiring that equations (5a) and (5b) are satisfied at all $N M$ collocation points given by equations ( $9 a$ ) and $(9 b)$. Refer to ref. [12] for evaluating the derivatives of Chebyshev polynomials. The pseudospectral algebraic eigenvalue problem is also solved using the EISPACK:RGG subroutine.

\section{NUMERICAL RESULTS}

Table 1 shows the convergence of the critical Rayleigh number $\left(R a_{c}\right)$ as the number of basis functions are increased for different solution methods. Note the lack of convergence of $R a_{c}$ when the algebraic system is obtained by taking inner products with the Chebyshev polynomials, i.e. we replace the first two basis functions in the integrands of equations (7) with $T_{k}(x) T_{i}(y)$. We do not fully understand why the con- 
Table 1. Convergence of the critical Rayleigh number (perfectly conducting side walls, $A=1$ )

\begin{tabular}{|c|c|c|c|c|}
\hline$N \times M$ & Galerkin & $\begin{array}{l}\text { Pseudo- } \\
\text { spectral }\end{array}$ & $\begin{array}{l}\text { Chebyshev } \\
\text { inner product }\end{array}$ & Velte $[8]$ \\
\hline $2 \times 2$ & 4971.575240 & 5010.7 & & \\
\hline $3 \times 3$ & 4947.244837 & 5021.5 & & \\
\hline $4 \times 4$ & 4946.860470 & 5011.6 & & \\
\hline $5 \times 5$ & 4946.874471 & 5011.7 & & 6660 \\
\hline $6 \times 6$ & 4946.873949 & 5011.7 & 10200 & \\
\hline $7 \times 7$ & 4946.873888 & & & \\
\hline $8 \times 8$ & 4946.873872 & & 5079 & 5290 \\
\hline $9 \times 9$ & $4946.87387 !$ & & & \\
\hline $10 \times 10$ & & & 5997 & \\
\hline $12 \times 12$ & & & & 5080 \\
\hline $14 \times 14$ & & & & 5030 \\
\hline
\end{tabular}

vergence for this method is so poor, but we suspect that the algebraic eigenvalue problem obtained by these methods does not retain the proper self-adjoint properties of the differential system. The property of marginal stability [9] indicates only real eigenvalues exist for the self-adjoint system. The Galerkin and pseudospectral methods show only real eigenvalues at this aspect ratio, as expected. But many spurious complex eigenvalues appear when the inner product is taken with Chebyshev polynomials and that destroys the accuracy of non-spurious eigenvalues [10], as shown here. The Galerkin and pseudospectral values appear to have converged to different values. We will demonstrate that the results from the Galerkin algorithm are more accurate.

Table 1 shows results when the truncation in both directions is the same, i.e. $N=M$. Most results, except those at high aspect ratio will be presented for this case. However, we find that the rcsolution in the $x$ direction is more important even at small aspect ratios. For example, for $A=1$ and $N=3, M=2$, the $R a_{\mathrm{c}}$ improves to 4949.2 compared to $R a_{c}=4969.6$ when $N=2, M=3$. For the high aspect ratio results $M$ need not be larger than 3 to obtain the results to the accuracy shown, while $N$ must be as high as 10 .

Figures 1-3 show the streamlines and isotherms at the onset of convection obtained by evaluating the Galerkin eigenfunction coefficients for various aspect ratios. Due to symmetry about $x=0$, we show only half the domain for the streamlines and isotherms. Figure 4, an enlargement of the lower corner of Fig. 1 , shows the sequence of recirculating corner eddies predicted by Moffatt [13]. Figure 4 clearly indicates the singular behavior at corners. Spectral methods [14] are exponentially convergent if the solution is infinitely differentiable. However, the Chebyshev spectral method converges so rapidly that six significant digit accuracy is obtained with $N=5$ for small aspect ratios and $N=6$ for aspect ratios up to $A=7$ (not shown).

To check the convergence of the critical eigenvalue, we assume that the exact eigenvalue is given using the Galerkin method, quadruple precision ( 32 digits), and $9 \times 9$ basis functions. The semi-log plot of Fig. 5 shows

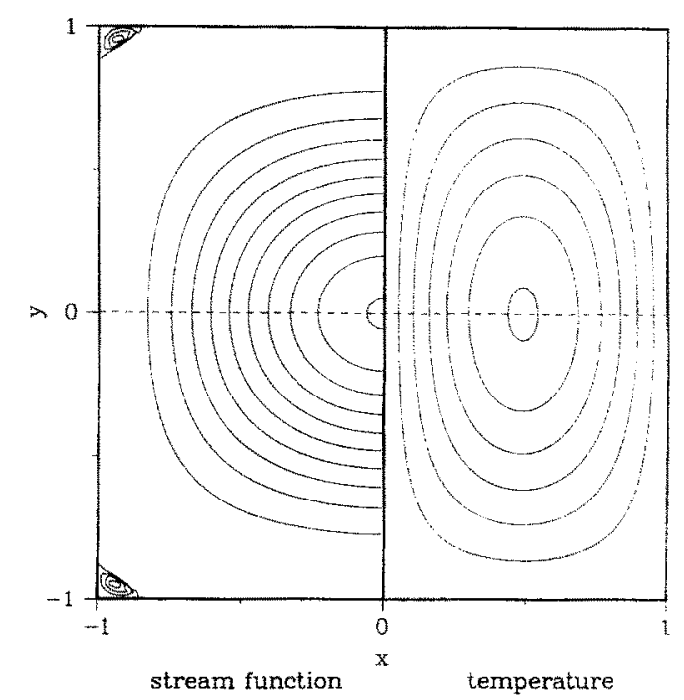

Fig. 1. Streamlines (left) and isotherms (right) for conducting side walls at $A=1$ and $R a_{c}=4046.8$. The streamlines are shown in increments of 5 except in the corners where the increment is $2 \times 10^{-4}$. The maximum streamline value is approximately 50 in the primary vortex. The isotherms are shown in increments of 0.5 .

that this error converges very rapidly using the spectral method as compared to Velte's [8] central secondorder finite difference method. Extrapolation of Velte's convergence data for $A=1$, reproduced in Table 1, gives an estimated converged value of $R a_{\mathrm{c}}=$ 4950. The convergence rate is then approximately $N^{-0.8}$ for his central second-order finite difference mesh compared to an apparent convergence rate greater than $N^{-10}$ for the Galerkin method.

The Galerkin method using double precision shows small oscillations when the number of basis function truncation is greater than $6 \times 6$. But the Galerkin method using quadruple precision shows no oscil-

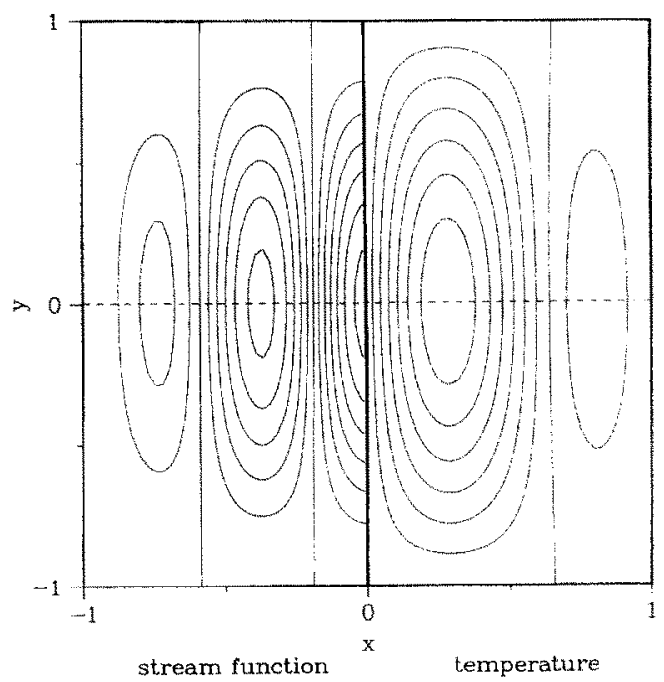

Fig. 2. Streamlines (left) and isotherms (right) for conducting side walls at $A=3$ and $R a_{\mathrm{c}}=1964.5$. The streamlines are shown in increments of 1 and isotherms are shown in increments of 0.3 . 


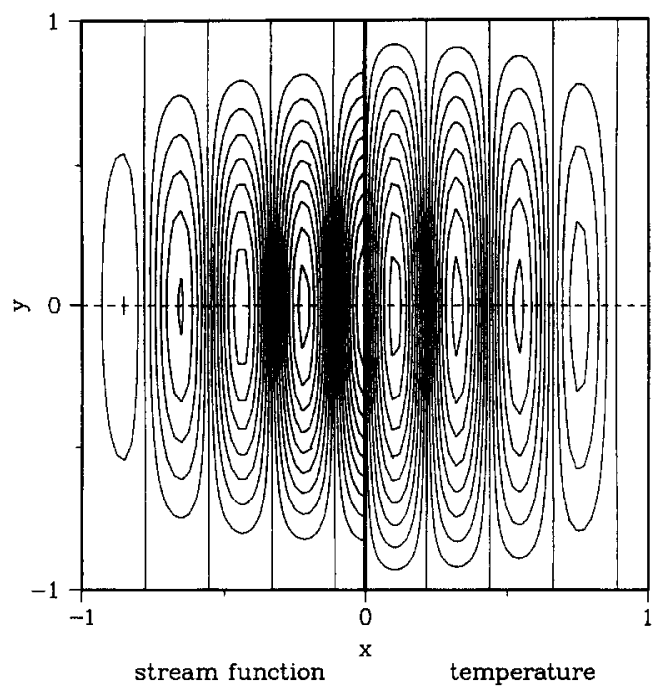

FIG. 3. Streamlines (left) and isotherms (right) for conducting side walls at $A=9$ and $R a_{\mathrm{c}}=1711.3$. The streamlines are shown in increments of 2 and isotherms are shown in increments of 0.3

lation and 9 significant digit accuracy is obtained with $N=M=8$. The precision error lies in the EISPACK algebraic eigenvalue routine. That is, if we enter matrix coefficients computed from double precision into the more precise eigenvalue routine, we would obtain the quadruple precision results shown in Table 1 and Fig. 5. However, we have found that we can replicate the quadruple precision results using double precision by normalizing the eigenmatrix such that the maximum coefficient in each row is one. Although eight digit accuracy is obtained without this procedure, it is worth noting that this procedure should be followed for highly accurate solutions even when the matrix is not ill-conditioned. In contrast, no improvement occurs for the pseudospectral or Cheby-

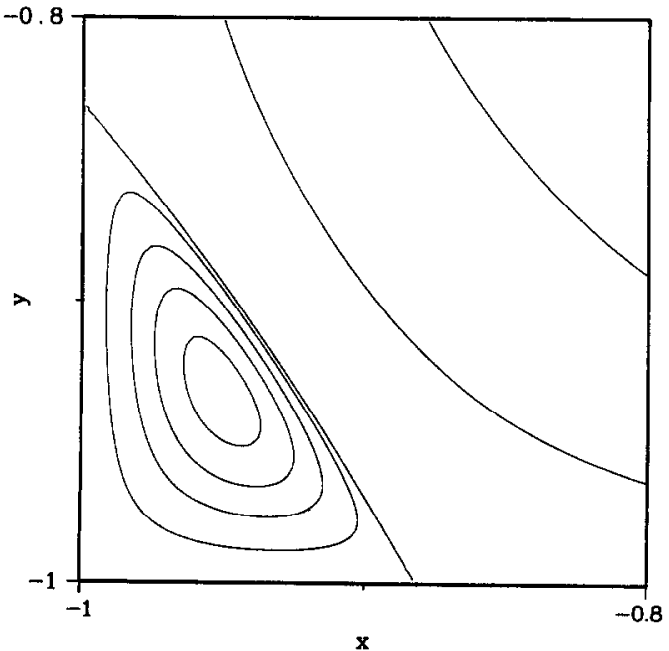

FIG. 4. Enlargement of Moffatt eddies at the corner for $A=1$ and $R a_{\mathrm{c}}=4046.8$. The streamline contours have the values $-0.1,-0.01,0,1 \times 10^{-4}, 3 \times 10^{-4}, 5 \times 10^{-4}$, and $7 \times 10^{-4}$.

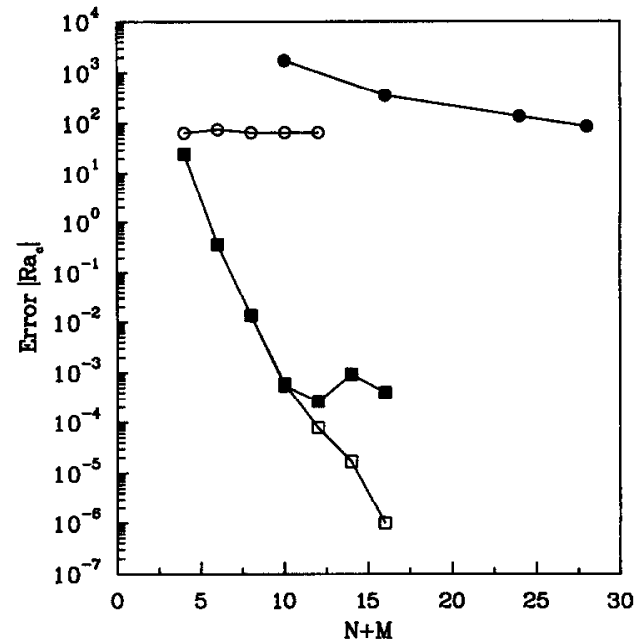

Fig. 5. Convergence of eigenvalue $R a_{\mathrm{c}}$ for $A=1$. $\square$, Galerkin method (quadruple precision); $\mathbf{E}$, Galerkin method (double precision);, central second-order finite difference method; $O$, pseudospectral method.

shev inner product methods when the same procedure is followed.

Haidvogel and Zang [15] show that the spectral coefficients decay algebraically as $(n+m)^{-6}$ for the Poisson equation, which has weak corner singularities of $r^{2} \log r$. For the special case of a right-angled corner, we can use symmetry arguments to show that the singularity of the Helmholtz-type equation we solve here is weaker, i.e. $r^{3} \log r$. Hence, the coefficients on a $\log -\log$ plot converge algebraically with order $(n+m)^{-8}$, as shown in Fig. 6. But the pseudospectral coefficients diverge when $n+m$ is approximately greater than 8 . To check for the unexpected divergence, we show the residual of the momentum equation (la) using the most unstable eigenfunction for the Galerkin and pseudospectral methods in Figs. 7-9.

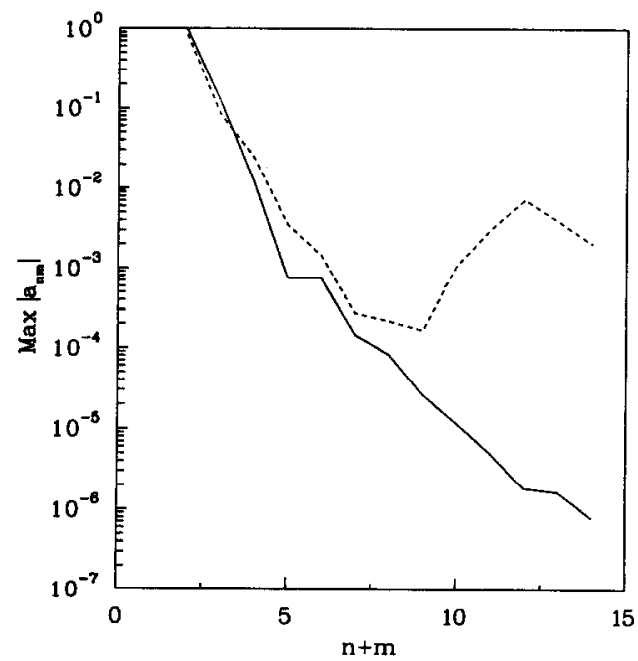

Fig. 6. Convergence of spectral and pseudospectral coefficients of the most unstable eigenfunction for $A=1$ and conducting side walls. Only the largest absolute value of the coefficient for fixed $n+m$ is plotted: - , Galerkin coefficients; -----, pseudospectral coefficients. 


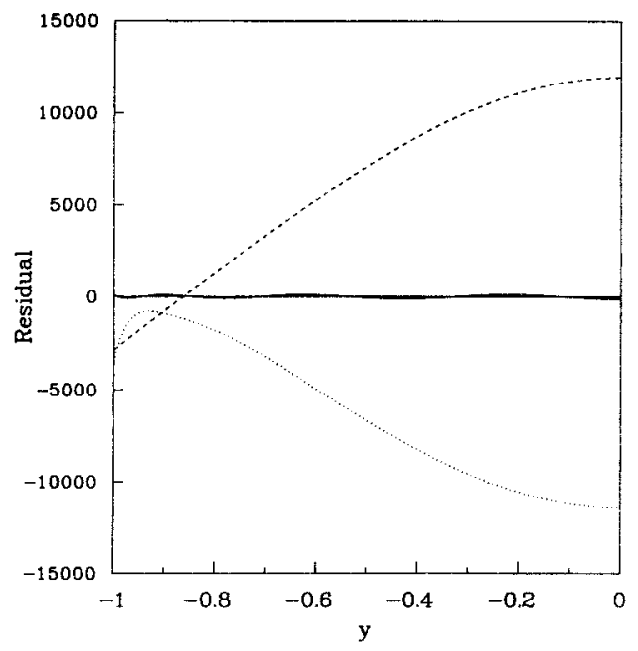

Fig. 7. Residual of the momentum equation for the pseudospectral and Galerkin approximations. The $x$ value is one of the collocation points:---.--, Galerkin approximation at $x=-0.11196$ (near core) $; \cdots$, , Galerkin approximation at $x=-0.99371$ (near wall); approximation at $x=-0.11196$ (near core).

Both methods are shown for $7 \times 7$ basis functions, $A=1$, and perfectly conducting walls. The residuals are shown for the interval $-1<y<0$ becausc of symmetry. The residuals, like the eigenfunctions, are only determined to within an arbitrary constant. To compare the two different methods, it is important to normalize the two results in the same way. We have done this as indicated by the same magnitude of the lowest order coefficients shown in Fig. 6. The pseudospectral calculations show zero residuals at collocation points, as expected (Figs. 7 and 8). These calculations are highly accurate for the core, especially near collocation points (Fig. 7), but produce large

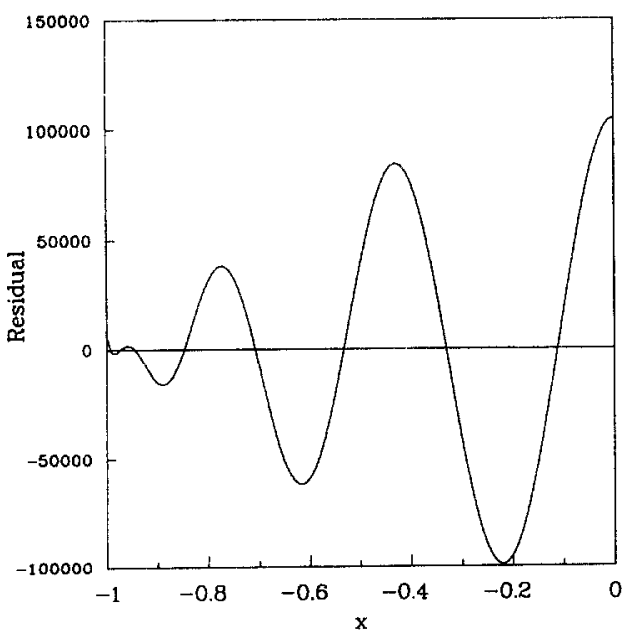

FIG. 8. Residual of the momentum equation for pseudospectral approximation. The $x$ value is one of the collocation points near the wall $(x=-0.99371)$ and $y$ varies within the interval $[-1,0]$. This residual is plotted separately from Fig.

7 since the scales vary by a factor of approximately 30 .

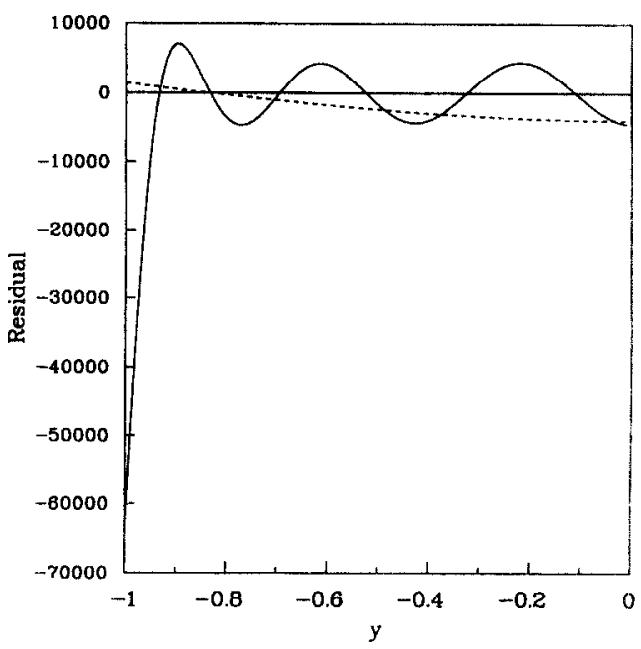

FIG. 9. Residual of the momentum equation for pseudospectral and Galerkin approximation. The $x$ value is between collocation points $(x=-0.6):-----$, Galerkin approximation; - , pseudospectral approximation.

Table 2. Critical Rayleigh number for perfectly conducting side walls

\begin{tabular}{crrr}
\hline Aspect ratio & Rolls & This work & Velte [8] \\
\hline 0.5 & 1 & 31973.4 & \\
$\frac{2}{3}$ & 1 & 12748.0 & 13380 \\
1.0 & 1 & 4946.8 & 5030 \\
1.3 & 1 & 3529.3 & 3510 \\
1.5 & 1 & 3145.9 & 3090 \\
2.0 & 2 & 2347.5 & 2350 \\
3.0 & 3 & 1964.5 & \\
4.0 & 4 & 1837.9 & \\
5.0 & 5 & 1780.5 & \\
6.0 & 6 & 1749.7 & \\
7.0 & 7 & 1732.7 & \\
8.0 & 8 & 1722.2 & \\
9.0 & 9 & 1711.3 & \\
\hline
\end{tabular}

Table 3. Critical Rayleigh number for perfectly insulated side walls

\begin{tabular}{cccc}
\hline Aspect ratio & Rolls & This work & Kurzweg [7] \\
\hline 0.5 & 1 & 12032.5 & 12120 \\
1.0 & 1 & 2552.97 & 2585.6 \\
2.0 & 2 & 1983.0 & 2015 \\
3.0 & 3 & 1841.3 & 2010 \\
4.0 & 4 & 1781.5 & \\
5.0 & 5 & 1750.2 & \\
6.0 & 6 & 1731.7 & \\
7.0 & 7 & 1719.5 & \\
\hline
\end{tabular}

errors near the walls (Figs. 8 and 9). The Galerkin method gives uniform and small residuals over the interval compared to the pseudospectral method (Figs. 7 and 9). We suspect that the poor convergence of the pseudospectral coefficients shown in Fig. 6 is caused by a Runge-like phenomenon near the walls [12].

Tables 2 and 3 summarize $R a_{\mathrm{c}}$ computed by the Galerkin method and the corresponding number of 


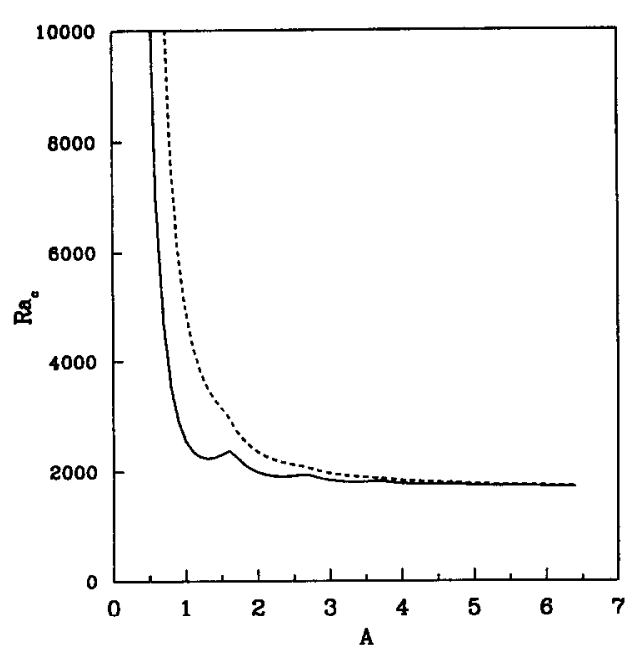

FIG. 10. Critical Rayleigh number stability as a function of aspect ratio: :----, conducting side walls; - , insulated side walls.

convective rolls for various aspect ratios. For aspect ratios less than 1.6 , the instability is a single convective roll. At an aspect ratio of 0.5 , the Rayleigh number of the first even mode for conducting side walls is 31 973.4, whereas the Rayleigh number for the first odd mode is 74465.7 . The transition in the number of rolls for conducting and insulated side walls occurs near aspect ratios of 1.6, 2.7, 3.7, 4.7, and 5.7. Figure 10 shows that the critical Rayleigh number for conducting side walls is always larger than the corresponding one for insulated side walls. The stability curves for either side wall boundary condition peak at the aspect ratios at which the number of rolls increases, alternating between even and odd rolls. Charlson and Sani [9] show the same trend in cylindrical layers of fluid heated from below.

At $A=2$, Velte [8] shows three rolls for the lowest eigenvalue $(R a=2350)$ and two rolls for the second lowest eigenvalue $(R a=2470)$. But our results show two rolls for the lowest eigenvalue ( $R a=2347.5)$ and three rolls for the second lowest eigenvalue $(R a=2570.3)$. At aspect ratios of 1.3 and 1.5 , our critical Rayleigh numbers are higher than those of Velte. Our Galerkin results converge to the ten digits shown on Table 1 with as little as $9 \times 9$ degrees of freedom and converge to the correct solution from above (i.e. they overpredict $R a_{c}$ ) as expected from the self-adjoint system.

We also note that our high aspect results seem to converge to that of the infinite layer $R a_{c}$ of 1707.8 with a 0.99217 aspect ratio of individual cells. The aspect ratio of the cells next to the walls adjusts to fit the enclosure geometry as shown in Fig. 3. Hence one can determine the stabilizing effects of the side walls for thin cavities to determine the suitability of using the more simple periodic boundary conditions. These large aspect ratio results would not be possible without a very efficient numerical scheme. We have obtained results for very large aspect ratios with $R a_{c}$ less than the periodic case. For example, for $A=21$ we obtain $R a_{\mathrm{c}}=1686.3$ and 1685.7 for conducting and insulating side walls, respectively. Further work is required since equation (8) is not formally self adjoint and hence can allow complex eigenvalues. We occasionally obtain complex eigenvalues when $A$ becomes large. Also, some computations for large $A$ do not converge from above as $N$ and $M$ are increased as expected from the Galerkin method. Hence, these smaller $R a_{\mathrm{c}}$ are suspect and it is not clear what physical mechanism could be enhancing the instability.

\section{CONCLUSIONS}

The Chebyshev spectral method as applied here is shown to be very accurate at determining the onset of two-dimensional natural convection in a box. When a full Galerkin method is used, six digit accuracy is obtaincd using as little as $5 \times 5$ basis functions for the temperature and stream function. Critical Rayleigh numbers are compared with those predicted by previous investigators. This appears to be an example where the corner singularity can be ignored while obtaining superb convergence to engineering accuracy [3] as long as the Galerkin method is used.

Very surprisingly, the pseudospectral approach failed for this eigenvalue problem. Although the eigenfunction problem is satisfied at the collocation points, the solution appears to 'resonate' with large residuals between collocation points, especially near the boundaries. These solutions do not converge. Similarly, forming the algebraic system using Chebyshev inner products rather than using basis functions that satisfy the boundary conditions also produce spurious eigenvalues and non-converging results. This is unfortunate, since the Galerkin method is the most difficult to implement. The failures of these other two methods are important to understand and further study is required. However, the extension of the Galerkin approach for more complex geometries using spectral elements or mapping should be straightforward.

Acknowledgements-This work was partially supported by the National Science Foundation Contracts MSM-8504456 and DMC 8716766 and partially by the Naval Research Laboratory Contract N00014-85-K-2019.

\section{REFERENCES}

1. S. A. Orszag, Accurate solution of the Orr-Sommerfeld stability equation, J. Fluid Mech. 50, 689-703 (1971).

2. A. Zebib, A Chebyshev method for solution of boundary value problems, J. Comp. Phys. 53, 443-455 (1984).

3. J. P. Boyd, An analytical and numerical study of the two-dimensional Bratu equation, J. Scient. Comp. 1, 183-206 (1986).

4. W. W. Schultz, A. Zebib, S. H. Davis and Y. Lee, Nonlinear stability of Newtonian fibers, J. Fluid Mech. 19, 455-475 (1984).

5. A. Schlüter, D. Lortz and F. H. Busse, On the stability of steady finite amplitude convection, J. Fluid Mech. 23, 129-144 (1965).

6. S. H. Davis, Convection in a box : linear theory, J. Fluid Mech. 30, 465-478 (1967). 
7. U. H. Kurzweg, Convective instability of a hydromagnetic fluid within a rectangular cavity, Int. J. Heat Mass Transfer 8, 35-41 (1965).

8. W. Velte, Stabilitätsverbalten und Verzweigung stationärer Lösungen der Navier-Stokesschen Gleichungen, Arch. Ration. Mech. Analysis 16, 97-124 (1964).

9. G. S. Charlson and R. L. Sani, Thermoconvective instability in a bounded cylindrical fluid layer, Int. J. Heat Mass Transfer 13, 1479-1496 (1970).

10. A. Zebib, Removal of spurious modes encountered in solving stability problems by spectral methods, J. Comp. Phys. 70, 521-525 (1987).

11. D. Gottlieb, M. Y. Hussaini and S. A. Orszag, Theory and applications of spectral methods. In Spectral Method for Partial Differential Equations, pp. 1-54. SIAM, Philadelphia (1984).

12. J. P. Boyd, Spectral Methods. Springer, Berlin (1989), in press.

13. H. K. Moffatt, Viscous and resistive eddies near a sharp corner, J. Fluid Mech. 18, 1-18 (1964).

14. D. Gottlieb and S. A. Orszag, Numerical analysis of spectral method: theory and applications, Soc. Ind. Appl. Math., Philadelphia (1977).

15. D. B. Haidvogel and T. Zang, The efficient solution of Poisson's equation in two-dimensions via Chebyshev approximation, J. Comp. Phys. 30, 167-180 (1979).

\section{APPENDIX}

Here we list the value of inner product for Chebyshev polynomials. Gottlieb and Orszag $[1,14]$ derive the following formulae using the identity and orthogonality of Chebyshev polynomials :

$$
\left(T_{m}, T_{n}\right)= \begin{cases}\frac{\pi}{2} & \text { if } n=m \neq 0 \\ \pi & \text { if } n=m=0 \\ 0 & \text { otherwise }\end{cases}
$$

$\left(T_{m}, \frac{\mathrm{d}}{\mathrm{d} x} T_{n}\right)=$

$$
\begin{cases}\pi n & \text { if } m=0,1, \ldots, M, n=m+1, m+3, \ldots, N \\ 0 & \text { otherwise }\end{cases}
$$

$$
\left(T_{m}, \frac{\mathrm{d}^{2}}{\mathrm{~d}^{2} x} T_{n}\right)=
$$

$$
\begin{cases}n\left(n^{2}-m^{2}\right) \frac{\pi}{2} & \text { if } m=0,1, \ldots, M, n=m+2, m+4, \ldots, N \\ 0 & \text { otherwise }\end{cases}
$$

$\left(T_{m}, \frac{\mathrm{d}^{4}}{\mathrm{~d}^{4} x} T_{n}\right)=$

$$
\left\{\begin{array}{l}
n\left[n^{2}\left(n^{2}-4\right)^{2}-3 m^{2} n^{4}+3 m^{4} n^{2}-m^{2}\left(m^{2}-4\right)^{2}\right] \frac{\pi}{48} \\
\text { if } m=0,1, \ldots, M, n=m+4, m+6, \ldots, N \\
0 \quad \text { otherwise. }
\end{array}\right.
$$

\title{
ETUDE DE LA STABILITE DU FLUIDE DANS UNE CAVITE RECTANGULAIRE PAR UNE METHODE SPECTRALE
}

Résumé-On étudie numériquement la stabilité hydrodynamique de la convection de Rayleigh dans une cavité rectangulaire. Les calculs supposent que le mouvement du fluide de Boussinesq est bidimensionnel. Les équations sont résolues par une méthode spectrale de Chebyshev. On doit faire attention dans l'obtention du problème propre algébrique équivalent pour éviter des racines fausses et des résultats erronées. Les nombres de Rayleigh critiques sont déterminés pour différents rapports de forme.

\section{ZUR STABILITÄT EINES FLUIDS IN EINEM RECHTECKIGEN HOHLRAUM NACH EINER SPEKTRALMETHODE}

Zusammenfassung - Es wird die hydrodynamische Stabilität der Rayleigh Konvektion in einem rechteckigen Hohlraum numerisch untersucht. Bei den Berechnungen wird die Fluidbewegung nach Boussinesq als zweidimensional angenommen. Die charakteristischen Gleichungen werden nach einer Chebyshev-Spektralmethode gelöst. Bei Aufstellung des äquivalenten algebraischen Eigenwertproblems muß aufgepaßt werden, um unechte Wurzeln und ungenaue Ergebnisse zu vermeiden. Für verschiedene Seitenverhältnisse werden kritische Rayleigh-Zahlen bestimmt.

\section{ИССЛЕДОВАНИЕ УСТОЙЧИВОСТИ ЖИДКОСТИ В ПРЯМОУГОЛЬНОЙ КАМЕРЕ СПЕКТРАЛЬНЫМ МЕТОДОМ}

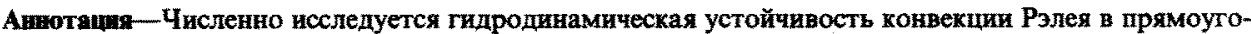
льной полости. Предполагается, что двкжение жидкости, опнсываемой в приближении Буссинеска, является двумерным. Характеристические уравнения решаются спектральным методом Чебышева. Во избехание появления ложных корней и неточных результатов обсуждаются подходы к построению эквивалентной алтебрацческой задачй на собственкые значения. Определскы критическе значения мисла Рэлея для разичных отношенй сторои полости.
\end{abstract}

